This item was submitted to Loughborough's Research Repository by the author.

Items in Figshare are protected by copyright, with all rights reserved, unless otherwise indicated.

\title{
Explosive voluntary torque is related to whole-body response to unexpected perturbations
}

PLEASE CITE THE PUBLISHED VERSION

https://doi.org/10.1016/j.jbiomech.2018.09.016

PUBLISHER

(C) Elsevier

VERSION

AM (Accepted Manuscript)

\section{PUBLISHER STATEMENT}

This paper was accepted for publication in the journal Journal of Biomechanics and the definitive published version is available at https://doi.org/10.1016/j.jbiomech.2018.09.016.

\section{LICENCE}

CC BY-NC-ND 4.0

\section{REPOSITORY RECORD}

Behan, Fearghal, Matthew T.G. Pain, and Jonathan P. Folland. 2019. "Explosive Voluntary Torque Is Related to Whole-body Response to Unexpected Perturbations". figshare. https://hdl.handle.net/2134/36239. 


\section{TITLE PAGE}

2

3 Title:

4 Explosive Voluntary Torque is Related to Whole-body Response to Unexpected 5 Perturbations

6 Authors:

7 Fearghal P. Behan ${ }^{1,2,3}$, Matt T.G. Pain ${ }^{2}$, Jonathan P. Folland ${ }^{1,2}$

8 Affiliations:

$9{ }^{1}$ Arthritis Research UK Centre for Sport, Exercise and Osteoarthritis, Loughborough 10 University, Leicestershire, UK.

$11{ }^{2}$ School of Sport, Exercise, and Health Sciences, Loughborough University, Leicestershire, 12 UK.

$13{ }^{3}$ Research Department, Aspetar Sports Medicine Hospital, Doha, Qatar.

\section{Corresponding Author:}

16 Fearghal P. Behan. School of Sport, Exercise, and Health Sciences, Loughborough

17 University, Leicestershire, UK, LE11 3TU. Email: F.Behan@lboro.ac.uk.

FB, MTGP and JPF all contributed to study design, data acquisition, analysis, interpretation

20 and article drafting and revising. All authors have read and approved the final submitted 21 manuscript.

23 Keywords:

24 Muscle function, dynamic balance, injury prevention, knee extensors, plantar flexors. 
Word Count: 3,025 words

27

\section{Abstract}

Explosive torque has been demonstrated to relate to static balance. However, sports injuries occur dynamically and unpredictably, yet the relationship between explosive torque and balance response to dynamic perturbations is unknown. This study investigated the relationship between explosive torque of the plantar flexors and knee extensors and the centre of mass $(\mathrm{COM})$ response to unexpected perturbations. Thirty-three healthy subjects (17 females, 16 males) were assessed for maximal and explosive isometric knee extension (KE) and plantar flexion (PF) torque and COM response (velocity (COMV), displacement (COMD)) to unexpected platform translations. Relationships between explosive torque and balance measures were investigated using Pearson's correlation and multiple regression. A negative relationship between PF explosive torque at 50, 100, and $150 \mathrm{~ms}$ and COMV at 300, 400 , and $500 \mathrm{~ms}(r=-0.363$ to $-0.508, p \leq 0.049)$, and COMD at 400 and $500 \mathrm{~ms}(r=-0.349$ to $0.416, p \leq 0.046)$ was revealed. A negative relationship between $\mathrm{KE}$ explosive torque at 50, 100, and $150 \mathrm{~ms}$ and COMV at $400 \mathrm{~ms}(r=-0.381$ to $-0.411, p \leq 0.029)$ but not COMD was also revealed. Multiple regression found PF 100 ms predicted $17.3 \%$ of variability in COMD at $500 \mathrm{~ms}$ and $25.8 \%$ of variability in COMV at $400 \mathrm{~ms}$. These results suggest that producing torque rapidly may improve $\mathrm{COM}$ response to unexpected perturbation. 
Introduction

53 Sporting participation has obvious physical, social and psychological benefits but contains

54 significant musculoskeletal injury risk (Bahr and Holm, 2003). An anterior cruciate ligament

55 (ACL) rupture is one of the most common and costly sports injuries (Domire et al., 2001). It

56 has been estimated that an ACL injury ages the knee 30 years and individuals who suffer a knee injury are at a more than 5-fold greater increase of developing osteoarthritis (Butler et al., 2008). Therefore, investigating potential contributors to injury mechanisms that may guide novel preventative strategies remain important.

60

ACL injuries occur within $50 \mathrm{~ms}$ of foot contact (Krosshaug et al., 2007) thus the ability to produce muscular force rapidly may be important in stabilising joints and preventing injurious positions (Zebis et al., 2010). The muscular ability to produce force rapidly is commonly referred to as explosive torque/force (i.e. at specific time points) or rate of torque/force development (RTD/RFD; i.e. gradient over specific periods). Explosive torque has been investigated in many populations (males, females, elderly, performance athletes) and demonstrated to be a determinant of athletic performance (Tillin et al., 2013; West et al., 2011). Post ACL repair, the ability to produce torque explosively has been demonstrated to predict hopping and vertical jump performance (Pua et al., 2017), to display larger asymmetries than maximal strength (Knezevic et al., 2014), and to be associated with selfreported knee function (Hsieh et al., 2014). However, there has been limited research into the relationship between explosive torque and balance performance. Explosive torque has been related to sway index $(r=-0.559, p=0.010)$ (Palmer et al., 2014) and RTD to balance measures in elderly subjects (Ema et al., 2016; Izquierdo et al., 1999]. RTD measures have also been found to predict tests that challenge lateral stability (Chang et al., 2005) and 
76 discriminate between elderly fallers and non-fallers (Bento et al., 2010, Pjinappels et al., 2008). Collectively these results suggest that the ability to produce torque explosively may be related to balance performance and falls prevention.

80 Sports injuries occur in dynamic, unpredictable situations and often involve unexpected 81 perturbations due to collision or impact (Krosshaug et al., 2007). Therefore, relationships between explosive force and static balance measures, such as sway index (Palmer et al., 2014), or premeditated balance tasks (Izquierdo et al., 1999) do not address the ability to control body position and balance in response to unexpected dynamic perturbations that occur within sports. Explosive torque has been demonstrated to improve dynamic balance recovery restricted to utilising the ankle strategy (Robinovitch et al., 2002) but has not been investigated with a whole-body response replicating the sporting environment. Accordingly, this study aimed to investigate whether knee extensor and plantar flexor explosive torque were related to the ability to stabilise the $\mathrm{COM}$ in response to unexpected perturbations. It was hypothesised that greater explosive muscular torque would be related to lower COM motions to unexpected perturbations.

\section{Methods}

\section{Participants}

96 Thirty-three healthy, young, participants (17 females: age $22.9 \pm 3.1 \mathrm{yr}$, height $1.66 \pm 0.06 \mathrm{~m}$, 97 mass $65.0 \pm 8.3 \mathrm{~kg} ; 16$ males: age $24.7 \pm 5.1$, height $1.81 \pm 0.09 \mathrm{~m}$, mass $81.1 \pm 9.5$ ) provided written informed consent prior to participating in this study, which was approved by the Loughborough University Ethical Approvals Committee. Participants had a BMI of $\leq 26$

$100 \mathrm{~kg} \cdot \mathrm{m}^{-2}$, no history of traumatic lower limb injury or current musculoskeletal condition and 
101 were not involved in systematic physical training. Body mass and height were measured 102 using a calibrated scale and stadiometer (Seca, Hamburg, Germany). All participants had

103 moderate levels of physical activity as assessed by the International Physical Activity 104 Questionnaire (iPAQ) (Craig et al., 2003). Participants were advised not to undertake any 105 unaccustomed/strenuous physical activity for 36 hours prior to testing and to have eaten and 106 drunk normally. To control for the possibility of menstrual cycle phase influencing 107 neuromuscular performance during testing females were required to have been taking the 108 combined monophasic oral contraceptive pill for $>6$ months and were tested between days 7 109 and 21 of pill consumption to minimize any fluctuations in endogenous gonadal hormones 110 (Pearson et al., 2011).

112 Study Design

113 This study used a cross-sectional study design. Participants visited the laboratory twice, 114 separated by 5-10 days, for a familiarisation session and a main trial. Sessions commenced

115 between 11.30am and 4.30pm. Familiarisation involved participants completing isometric 116 contractions of the plantar flexors $(\mathrm{PF})$ on an adapted commercial dynamometer and knee 117 extensors (KE) on a custom built isometric strength testing chair; followed by familiarisation 118 with dynamic perturbation testing. The main trial involved the same muscle function tasks of 119 both legs unilaterally, first PF and then KE measurements, followed by assessment of the 120 whole-body perturbation response to unexpected platform movements.

Muscle Function Measurements

123 Plantar Flexion: Participants were secured in an adapted commercial dynamometer (Con-trex,

124 Dubendorf, Switzerland) in a kneeling position with a custom upright to support the anterior 125 thigh, lock the proximal shank and minimise extraneous movement (Appendix 1A). The 
126 dynamometer fulcrum was positioned in line with the lateral malleolus and the ankle was

127 fixed at $90^{\circ}$ and the knee at $120^{\circ}\left(180^{\circ}=\right.$ full extension $)$. Three ankle straps were secured

128 tightly with a hip strap to further minimise any extraneous movements. The torque signal

129 from the dynamometer was amplified (x1000) and sampled at $2000 \mathrm{~Hz}$ using an external A/D

130 converter (Micro 1401; CED, Cambridge, UK) interfaced with a PC running Spike 2 software

131 (CED Ltd., Cambridge, UK). In offline analysis, torque data were low-pass filtered at $500 \mathrm{~Hz}$

132 using a fourth-order zero-lag Butterworth filter.

133

134 Knee Extension: Participants were secured in a customised dynamometer (Maffiuletti et al., 135 2016) with hip and knee joint angles of $125^{\circ}$ and $115^{\circ}$ respectively (Appendix 1B).

136 Adjustable strapping across the pelvis and shoulders prevented extraneous movement. A strap, $40 \mathrm{~mm}$ width of reinforced canvas webbing, was placed proximal to the ankle $(\sim 15 \%$ of tibial length above medial malleolus) in series with a calibrated S-beam strain gauge (Force Logic, Swallowfield, UK). The analogue force signal from the strain gauge was amplified

140 (x370) and sampled at $2000 \mathrm{~Hz}$ using an external A/D converter (Micro 1401; CED, 141 Cambridge, UK) interfaced with a PC running Spike 2 software (CED Ltd., Cambridge, UK).

142 In offline analysis, force data were low-pass filtered at $500 \mathrm{~Hz}$ using a fourth-order zero-lag

143 Butterworth filter, gravity corrected by subtracting baseline force, and multiplied by lever 144 length (distance from ankle strap centre to knee joint space) to calculate torque (Balshaw et 145 al., 2016).

147 Protocol

148 Maximum Voluntary Isometric Contractions: Participants performed a series of warm-up 149 contractions at $\sim 50 \%$ and $\sim 75 \%$ of perceived maximum voluntary torque (MVT) followed by 150 three maximum voluntary contractions (MVCs) each lasting 3s with $>30 \mathrm{~s}$ rest between. 
151 Participants were instructed to contract as hard as possible and motivated with a standardised script of verbal encouragement and real-time visual biofeedback of the force response of each contraction. MVT was defined as the greatest torque during any MVC or explosive

154 contraction.

156 Explosive Voluntary Isometric Contractions: Following a 2-minute rest post MVCs, 157 participants completed 10 explosive KE and PF contractions, separated by 20s. They were instructed to contract 'as fast and as hard as possible' for 1s with 'fast' emphasized. To provide biofeedback on their explosive performance the slope of the force time curve was

160 displayed throughout the contractions, with the peak slope of their best attempt highlighted.

161 Contractions associated with pre-tension or a counter movement were discarded and repeated. A visual marker on screen depicted $80 \%$ MVT during the explosive contractions and participants were instructed to achieve this level in order to ensure a forceful contraction.

164 Explosive contractions were performed until 10 contractions met criteria. Torque onset was

165 defined manually by visual identification by one trained investigator; this involved viewing 166 torque on an $\mathrm{x}$ axis scale of $300 \mathrm{~ms}$ prior to contraction and on a y axis scale of 0.68 N.m, 167 before zooming in to determine the instant of the final trough or peak before the signal deflected away from the envelope of baseline noise (Balshaw et al., 2016). Explosive voluntary torque (EVT) was measured at discrete time points: 50, 100, and $150 \mathrm{~ms}$ from

170 torque onset during the explosive contractions (e.g. PF 50, PF 100 etc.). All torque measures were normalised to body mass $(\mathrm{kg})$ for correlations and regression analysis.

Perturbation Response Testing

174 Perturbation trials were completed on a CAREN system (Computer Assisted Rehabilitation

175 Environment, Motek Medical, Amsterdam, Netherlands), a computer controlled Stewart 
176 platform that can independently perturb the support surfaces in each of the six degrees of

177 freedom. It has been found to be highly reliable for use in postural and balance research. ${ }^{19}$

178 Prior to the perturbations 57 spherical markers of $14 \mathrm{~mm}$ diameter were attached to the 179 participants to facilitate collection of kinematic data at $200 \mathrm{~Hz}$ using 9 T20 Vicon (Vicon,

180 Oxford Metrics Group, UK) cameras. Whole-body segmental anthropometric measurements

181 involved 45 measurements of each participant according to a reduced set inertial model

182 (Yeadon, 1990). These were utilised to calculate participant-specific segmental inertial

183 parameters for 15 body segments and used in the determination of joint centre locations and

184 segmental and whole-body COM.

185

186 The COM position was defined relative to the centre of the platform, specifically the mean 187 position of 4 markers located at the corners of the force plate. Participants were instructed to stand upright on the CAREN platform with hands by their side, the non-standing foot in a standardised position, the forefoot lightly touching the standing ankle, and asked to try to

190 remain stationary and not to take a step in response to the perturbation. The perturbation

191 commenced at random after participants had confirmed they were stable. Single leg standing was chosen to provide a sufficient balance stimulus. Four experimental conditions, sequentially in the following order: right foot/eyes open, left foot/eyes open, right foot/eyes closed, left foot/eyes closed, were completed with 16 trials per experimental set, resulting in 64 trials per subject. The direction order of each perturbation (left, right, forward, backward) was block randomised so that 4 of each direction occurred in each experimental set. Only anterior platform displacements were analysed (including eyes open and closed) to assess sagittal plane muscle function, with the other directions interspersed to reduce predictability. This resulted in 16 anterior trials per participant, with the average of these trials used for analysis. The perturbations were controlled by a custom script written in the Motek Medical 
D-Flow software. Anterior platform perturbations had a magnitude of $0.1 \mathrm{~m}$ and a maximum velocity of $0.3 \mathrm{~m} \cdot \mathrm{s}^{-1}$ (Figure 1). The order of platform directional perturbations was selected randomly during pilot testing and was consistent for all participants. Data were low pass filtered with a fourth-order zero-lag Butterworth filter using a cut off frequency of $15 \mathrm{~Hz}$. Initiation of perturbation was defined as when the velocity of the platform markers exceeded $0.005 \mathrm{~m} \cdot \mathrm{s}^{-1}$. COM displacement and velocity (over $5 \mathrm{~ms}$ time periods) were obtained from the initiation of perturbation to $500 \mathrm{~ms}$ post perturbation using Visual3D software (C-motion, Germantown, MD, USA) with values at $100 \mathrm{~ms}$ intervals used for analysis. COM displacement (COMD) after the onset of the perturbation was measured relative to the initial

210 position at onset of perturbation (defined as 0 ), and COM velocity (COMV) was defined as 211 instantaneous velocity at each frame.

\section{Statistical Analysis}

216 Average measures across right and left legs were utilised for statistical robustness. The 217 relationship of $\mathrm{KE}$ and $\mathrm{PF}$ MVT and EVT (at time points 50, 100, and $150 \mathrm{~ms}$ after contraction onset) with COMV and COMD values (100 ms intervals up to $500 \mathrm{~ms}$ ) were investigated using Pearson's correlation coefficients. Subsequently stepwise multiple regressions were undertaken to examine the influence of the entered predictor variables

221 (isometric strength parameters that displayed highest correlations with criterion variables) that independently explained a proportion of the total variance of COMD and COMV (criterion variables). Between subject coefficient of variation (CV) was obtained by

224 calculating the ratio of the standard deviation to the mean. Statistical significance was 
defined as $p<0.05$. All statistical procedures were performed with IBM SPSS Statistics for Windows (Version 22.0, NY, USA, IBM Corp.).

227

\section{Results}

Descriptive Data for Torque and Perturbation Measures

230 EVT increased progressively throughout the first $150 \mathrm{~ms}$ of explosive contractions for both muscle groups: KE EVT rose from 0.65 N.m.kg ${ }^{-1}$ at $50 \mathrm{~ms}(\mathrm{CV} 55 \%)$ to $2.26 \mathrm{~N} \cdot \mathrm{m}^{\mathrm{kg}} \mathrm{kg}^{-1}$ at 150 $\mathrm{ms}(\mathrm{CV} 32 \%)$ and PF EVT increased from 0.38 N.m.kg ${ }^{-1}$ at $50 \mathrm{~ms}(\mathrm{CV} 42 \%)$ to 1.82 N.m.kg${ }^{1}$ at $150 \mathrm{~ms}(\mathrm{CV} 23 \%$; Figure 2).

235 During the first $500 \mathrm{~ms}$ of anterior perturbations, the COMD relative to the base of support 236 (the platform) at onset was increasingly posterior (0.005 $\mathrm{m}$ at $100 \mathrm{~ms}$ (CV 23\%) up to $0.07 \mathrm{~m}$ 237 after $500 \mathrm{~ms}$ (CV 15\%), positive displacement value indicates posterior COMD). 238 Consequently, there was an increasing posterior COMV during the initial phase of perturbation, on average peaking $300 \mathrm{~ms}$ after perturbation (CV 13\%), before decreasing (decelerating) and becoming an anterior velocity after $500 \mathrm{~ms}$ (Figure 3).

PF EVT at all time points exhibited weak to moderate correlations with COMD in the later

244 phase of the perturbation (PF $50 \mathrm{~ms}$ and COMD at $500 \mathrm{~ms}, r=-0.380, p=0.029$; PF $100 \mathrm{~ms}$ and COMD 400-500 ms, $r=-0.349$ to $-0.416, p \leq 0.046$, Figure 4A; PF $150 \mathrm{~ms}$ and COMD

246 from $400-500 \mathrm{~ms}, r=-0.352$ to $-0.406, p \leq 0.044$, Figure $5 \mathrm{~A})$. PF EVT at all time points also exhibited weak to moderate negative correlations with COMV in the later phase of the 
248 perturbation (PF $50 \mathrm{~ms}$ and COMV 400- $500 \mathrm{~ms}, r=-0.421$ to $-0.459, p \leq 0.015$; PF $100 \mathrm{~ms}$ 249 and COMV 400- $500 \mathrm{~ms}, r=-0.398$ to $-0.508, p \leq 0.022$, Figure 4B; PF $150 \mathrm{~ms}$ and COMV $250300-500 \mathrm{~ms}, r=-0.346$ to $-0.479, p \leq 0.049$; Figure 5B). In contrast, PF MVT was unrelated 251 to either COMV or COMD ( $r=-0.053$ to $-0.246, p \geq 0.167)$.

Bivariate Correlations of KE Torque with COMD and COMV

KE EVT at 50, 100, and $150 \mathrm{~ms}$ showed weak to moderate negative correlations with COMV at $400 \mathrm{~ms}(r=-0.381$ to $-0.411, p \leq 0.021$, Figure $6 \mathrm{~A})$ but not with any measures of COMD (Figure 6B). KE MVT was negatively correlated with COMV at $300 \mathrm{~ms}(r=-0.371, p=$ 0.033) but not with COMD ( $r=-0.199$ to $-0.288, p \geq 0.104)$.

Multiple Regression Analysis

260 The muscle function measurements that were the strongest predictors of perturbation

261 response for each muscle group (PF 100 and KE 100) from the correlation analysis were 262 subsequently included in a stepwise multiple linear regression analysis. PF 100 predicted $26317.3 \%$ of the variability in COMD at $500 \mathrm{~ms}$ and $25.8 \%$ of the variability in COMV at 400 264 ms. KE 100 was not found to be a significant predictor of COMD or COMV within the 265 multiple regression analysis.

\section{Discussion}


268 The present study investigated the relationship between the COM stabilisation 269 (displacement/velocity) in response to unexpected anterior perturbations with both KE and 270 PF explosive torque. PF EVT had weak to moderate correlations with COMD from 400-500 $271 \mathrm{~ms}(r=-0.346$ to -0.508$)$ and COMV from $300-500 \mathrm{~ms}(r=-0.349$ to -0.416 ; i.e. more 272 explosive torque less COMD and COMV), with weaker correlations between KE EVT and 273 COMV at $400 \mathrm{~ms}(r=-0.381$ to -0.411$)$ but not with COMD. In contrast MVT of the PF and 274 KE were unrelated to COMD and unrelated/ weakly related to COMV. Moreover, the regression analysis revealed PF 100 to be the only neuromuscular variable to independently contribute to the explained variance in COMD and COMV.

278 These findings suggest that greater explosive torque, particularly from the distal (PF) musculature, results in better control of the COM in response to unexpected perturbations. PF EVT explained up to $25.8 \%$ of the variability in the COMD following perturbation. Thus, while PF EVT was the primary neuromuscular determinant of the ability to respond to unexpected perturbation, the majority of the variance remained unexplained. Somatosensory and reflex responses might also be expected to contribute substantially to the perturbation response. Nonetheless, in the current study significant correlations were consistently found for explosive torque measured at a range of time points, with both COMV and COMD from 300-500 ms indicating a genuine, systematic relationship.

288 Our findings of a negative relationship between explosive torque and COM response substantiates previous research that found higher explosive torque to be related to superior static or premeditated balance responses (Ema et al., 2016; Izquierdo et al., 1999; Jakobsen et

291 al., 2011; Palmer et al., 2014; Sundstrup et al., 2010). In overview, there is accumulating 292 evidence that lower limb explosive torque production may improve the ability to recover 
293 balance and appears to be an important component of falls risk and injury prevention (Bento

294 et al., 2010; Jakobsen et al., 2011; Sundstrup et al., 2010).

295

296 Repeated significant correlations were found between PF EVT and both COMV and COMD

297 providing evidence of a consistent relationship, however, KE EVT only correlated with

298 COMV at $400 \mathrm{~ms}$ and not with any COMD values, presumably because the KE did not 299 sufficiently influence COMV to effect COMD. Furthermore, PF EVT was the only predictor 300 of COM response in stepwise regression. Therefore, PF explosive torque appears more 301 important in controlling dynamic perturbation response. The greater importance of the PF 302 may be a consequence of a distal to proximal muscle activation pattern in response to 303 perturbations, where postural muscle activation begins at the base of support (Alexander et 304 al., 1992; Jakobsen et al., 2011; Sveistrup et al., 1997). A greater contribution of the ankle musculature and a lesser contribution of the knee musculature in achieving postural control in response to perturbation is in accordance with previous literature (Hall and Jensen, 2002;

307 Jakobsen et al., 2011).

308

309 In contrast to PF explosive torque PF MVT was not correlated with either COMD or COMV.

310 Other studies have also found weaker or no relationship for MVT and balance performance,

311 compared to EVT (Bento et al., 2010; Izquierdo et al., 1999; Lee et al., 2009). As maximal

312 torque can take more than $300 \mathrm{~ms}$ to produce it seems that explosive torque may be more

313 pertinent in response to rapid stimuli such as unexpected perturbations and sports related 314 injury mechanisms (Krosshaug et al., 2007; Sundstrup et al., 2010). The relationship between

315 EVT, and not MVT, to perturbation response demonstrates that explosive torque production,

316 rather than maximal torque, appears the most important neuromuscular characteristic in 317 responding to rapid alterations to postural balance. Therefore, future interventions for 
318 improving postural balance response may benefit from including specifically explosive 319 strength training, rather than training for maximal strength.

321 Correlations between $\mathrm{PF} / \mathrm{KE}$ explosive torque and $\mathrm{COM}$ response to perturbation only 322 became significant from $300 \mathrm{~ms}$ onwards. The anterior platform perturbation caused the

323 COM to initially move posteriorly relative to the base of support/platform (Nashner et al., 324 1979), before participants initiated a response to prevent the COM moving outside, and in 325 this case posterior to, the base of support (Lin and Woolacott, 2002). Considering muscle 326 response latency, potentially over $100 \mathrm{~ms}$ (Nashner et al., 1979) including sensory and 327 neurological delays, followed by electromechanical delay (Blenkinsop et al., 2016), it is 328 feasible that the initiation of compensatory muscular torque only commenced $150 \mathrm{~ms}$ after the perturbation. Thereafter muscular torque would have progressed rapidly to slow and ultimately reverse the posterior velocity of the COM imposed by the anterior platform displacement. This potential reversal of COMV consequently exhibited a reduction in COMD

332 from $400 \mathrm{~ms}$; implying reactive torque may have reduced the COM displacement from the 333 perturbing mechanism.

Many sporting injuries, such as ACL injuries, are in response to an activity involving an initial contact, such as landing and changing direction (Krosshaug et al., 2007). Therefore,

337 future research should attempt to replicate the current findings in a sporting specific scenario 338 to confirm the relationship between explosive torque and kinematic responses following a 339 task such as landing from a jump or changing direction. The current investigation may have 340 been limited by utilising isometric contractions, while facilitating controlled measurements of 341 maximum and explosive torque, this measurement may lack ecological validity to dynamic 342 functional movement. The current findings would also have been more robust with a larger 
343 number of participants. In conclusion, the ability to produce torque rapidly in the PF was

344 moderately associated with the response to unexpected perturbations, predicting up to $26 \%$ of

345 perturbation response. This indicates that higher explosive torque capacity, which can be 346 developed through training (Balshaw et al., 2016), may be useful in responding to external

347 collisions and disturbances of balance, and would be expected to reduce injury risk in 348 sporting situations.

Acknowledgements

351 Funding received from the Arthritis Research UK Centre for Sport, Exercise and 352 Osteoarthritis.

\section{Conflict of interest statement}

This is to confirm the authors of this work have no conflicts of interest.

\section{References}

1. Alexander, N.B., Shepard, N., Ju Gu, M., Schultz, A. 1992. Postural control in young and elderly adults when stance is perturbed: Kinematics. J. Gerontol. 47, 79-87.

2. Bahr, R., Holme, I. 2003. Risk factors for sports injuries-a methodological approach. Br. J. Sports Med. 37, 384-392.

3. Balshaw, T.G., Massey, G.J., Maden-Wilkinson, T.M., Tillin, N.A., Folland, J.P. 2016. Training-specific functional, neural, and hypertrophic adaptations to explosivevs sustained- contraction strength training. J. Appl. Physiol. 120, 1364-1373

4. Bento, P.C., Pereira, G., Ugrinowitsch, C., Rodacki, A.L. 2010. Peak torque and rate of torque development in elderly with and without fall history. Clin. Biomech. 25, 450-454. 
5. Blenkinsop, G.M., Pain, M.T.G., Hiley, M.J. 2016 Evaluating feedback time delay during perturbed and unperturbed balance in handstand. Hum. Mov. Sci. 4, 112-120.

6. Butler, R.J., Minick, K.I., Ferber, R., Underwood, F.B. 2008. Gait mechanism following ACL reconstruction: implications for the early onset of knee osteoarthritis. Br. J. Sports Med. 43, 366-370.

7. Chang, S.H.J., Mercer, V.S., Giuliaini, C.A., Sloane, P.D. 2005. Relationship between hip abductor rate of force development and mediolateral stability in older adults. Arch. Phys. Med. Rehabil. 86, 1843-1850.

8. Craig, C.L., Marshall, A.L., Sjostrom, M., Bauman, A.E., Booth, M.L., Ainsworth, B.E., Pratt, M., Ekelund, U., Yngve, A., Sallis, J.F., Oja, P. 2003. International physical activity questionnaire: 12-country reliability and validity. Med. Sci. Sports Exerc. 35, 1381-1395.

9. Domire, Z.J., Boros, R.L., Hashemi, J. 2001. An examination of possible quadriceps study. J. Biomech. 44, 1630-1632.

10. Ema, R., Saito, M., Ohki, S., Takayami, H., Yamada, Y., Akagi, R. 2016. Association between rapid force production by the plantar flexors and balance performance in elderly men and women. Age. 38, 475-483.

11. Hall, C.D., Jensen, J.D. 2002. Age-related differences in lower extremity power after support surface perturbations. J. Am. Geriatr. Soc. 50, 1782-1788.

12. Hsieh, C.J., Indelicato, P.A., Moser, M.W., Vandenborne, K., Chmielewski, T.L. 2015. Speed, not magnitude, of knee extensor torque production is associated with self-reported knee function early after anterior cruciate ligament reconstruction. Knee Surg. Sports Traumatol. Arthrosc. 23, 3214-3220. 

and explosive force production capacity and balance performance in men of different ages. Eur. J. Appl. Physiol. Occup. Physiol. 79, 260-267.

14. Jakobsen, M.D., Sundstrup, E., Krustrup, P., Aagaard, P. 2011. The effect of recreational soccer training and running on postural balance in untrained men. Eur. J. Appl. Physiol. 111, 521-530.

15. Knezevic, O.M., Mirkov, D.M., Kadija, M., Nedeljkovic, A., Jaric, S. 2014. Asymmetries in explosive strength following anterior cruciate ligament reconstruction. The Knee. 21, 1039-1045.

16. Krosshaug, T., Slauterbeck, J.R., Engebretesen, L., Bahr, R. 2007. Biomechanical analysis of anterior cruciate ligament injury mechanisms: three-dimensional motion reconstruction from video sequences. Scand. J. Med. Sci. Sports. 17, 508-519.

17. Lee, H.M., Cheng, C.K., Liau, J.J. 2009. Correlation between proprioception, muscle strength, knee laxity, and dynamic standing balance in patients with chronic anterior cruciate ligament deficiency. The Knee. 16, 387-91.

18. Lees, A., Vanrenterghem, J., Barton, G., Lake, M. 2007. Kinematic response 408 characteristics of the CAREN moving platform system for use in posture and balance research. Med. Eng. Phys. 29, 629-635.

19. Lin, S.I., Woollacott, M.H. 2002. Postural muscle responses following changing balance threats in young, stable older, and unstable older adults. J. Mot. Behav. 34, $37-44$.

20. Maffiuletti, N.A., Aagaard, P., Blazevich, A.J., Folland, J., Tillin, N.A., Duchateau, J. 2016. Rate of force development: physiological and methodological considerations. Eur. J. Appl. Physiol. 116, 1091-1116. 
21. Moore, S.P., Rushmer, D.S., Windus, S.L., Nashner, L.M. 1988. Human automatic postural responses: responses to horizontal perturbations of stance in multiple directions. Exp. Brain Res. 73: 648-658.

22. Nashner, L.M., Woollacott, M., Tuma, G. 1979. Organizations of rapid responses to postural and locomotor-like perturbations of standing man. Exp. Brain Res. 36, 463476.

23. Palmer, T.B., Hawkey, M.J., Thiele, R.M., Conchola, E.C., Adams, B.M., Akehi, K., Smith, D.B., Thompson, B.J. 2014 The influence of athletic status on maximal and rapid isometric torque characteristics and postural balance performance in Division 1 female soccer athletes and non-athlete controls. Clin. Physiol. Funct. Imaging. 35, 314-322.

24. Pearson, S.J., Burgess, K.E., Onambélé, G.L. 2011. Serum relaxin levels affect the in vivo properties of some but not all tendons in normally menstruating young women. Exp. Physiol. 96, 681-688.

25. Pijnappels, M., van der Burg, P.J., Reeves, N.D., van Dieen, J.H. 2008 Identification of elderly fallers by muscle strength measures. Eur. J. Appl. Physiol. 102, 585-592.

26. Pua, Y.H., Mentiplay, B.F., Clark, R.A., Ho, J.Y. 2017. Associations among quadriceps strength and rate of torque development 6 weeks post anterior cruciate ligament reconstruction and future hop and vertical jump performance: A prospective cohort study. J. Orthop. Sports Phys. Ther. 47, 845-852.

27. Robinovitch, S.N., Heller, B., Lui, A., Cortez, J. 2002. Effect of strength and speed of torque development on balance recovery with the ankle strategy. J. Neurophysiol. 88, 613-620.

28. Sundstrup, E., Jakobsen, M.D., Andersen, J.L., Randers, M.B., Petersen, J., Suetta, C., Aagaard, P., Krustrup, P. 2010. Muscle function and postural balance in lifelong 
trained male footballers compared with sedentary elderly men and youngsters. Scan.

442

443

444

445

446

447

448

449

450

451

452

453

454

455

456

457

458

459

460
J. Med. Sci. Sports. 20, 90-97.

29. Sveistrup, H., Woollacott, M.H. 1997 Practice modifies the developing automatic postural response. Exp. Brain Res. 114, 33-43.

30. Tillin, N.A., Pain, M.T.G., Folland, J.P. 2013 Explosive force production during isometric squats correlates with athletic performance in rugby union players. J. Sports Sci. 31, 66-76.

31. West, D.J., Owen, N.J., Jones, M.R., Bracken, R.M., Cook, C.J., Cunningham, D.J., Shearer, D.A., Finn, C.V., Newton, R.U., Crewther, B.T., Kilduff, L.P. 2011. Relationships between force-time characteristics of the isometric midthigh pull and dynamic performance in professional rugby league players. J. Strength Cond. Res. 25, 3070-3075.

32. Yeadon, M.R. 1990. The simulation of aerial movement: II. A mathematical inertial model of the human body. J Biomech. 23(1):67-74.

33. Zebis, M.K., Andersen, L.L., Ellingsgaard, H., Aagaard, P. 2010. Rapid hamstring/quadriceps force capacity in male vs female elite soccer players. J. Strength Cond. Res. 25, 1989-1993. 


\section{List of Figures}

Figure 1. Platform trajectory during an anterior perturbation showing (A) anterior displacement and (B) velocity.

Figure 2. Explosive knee extensor (A, black squares) and plantar flexor (B, black circles) torque for each measured time point $(\mathrm{ms})$. Values are mean $\pm \mathrm{SD}(\mathrm{n}=33)$ with maximum and minimum values (dashed line).

Figure 3. Centre of mass (COM), (relative to centre of moving force plate) Displacement (A, black squares) and COM Velocity (B, black circles) for each time point (ms) post perturbation. Values are mean $\pm \mathrm{SD}(\mathrm{n}=33)$ with maximum and minimum values (dashed line). Positive values indicate posterior displacement/velocity.

Figure 4. Sample scatter plots of PF explosive torque at $100 \mathrm{~ms}$ and (A) COM Displacement at $400 \mathrm{~ms}$ and (B) COM Velocity at $400 \mathrm{~ms}(\mathrm{n}=33)$.

Figure 5. Pearson's product moment correlation coefficients between explosive torque of the plantar flexors (PF) at three time points $(50,100,150)$ and centre of mass displacement $(\mathrm{COMD}, \mathrm{A})$ and centre of mass velocity $(\mathrm{COMV}, \mathrm{B})$ post perturbation $(\mathrm{n}=33)$. $\mathrm{Y}$ axis inverted for ease of visualization.

Figure 6. Pearson's product moment correlation coefficient between explosive torque of the knee extensors $(\mathrm{KE})$ at three time points $(50,100,150)$ and centre of mass displacement (COMD) (A) and centre of mass velocity (COMV) (B) post perturbation $(\mathrm{n}=33)$. Y axis inverted for ease of visualization. 

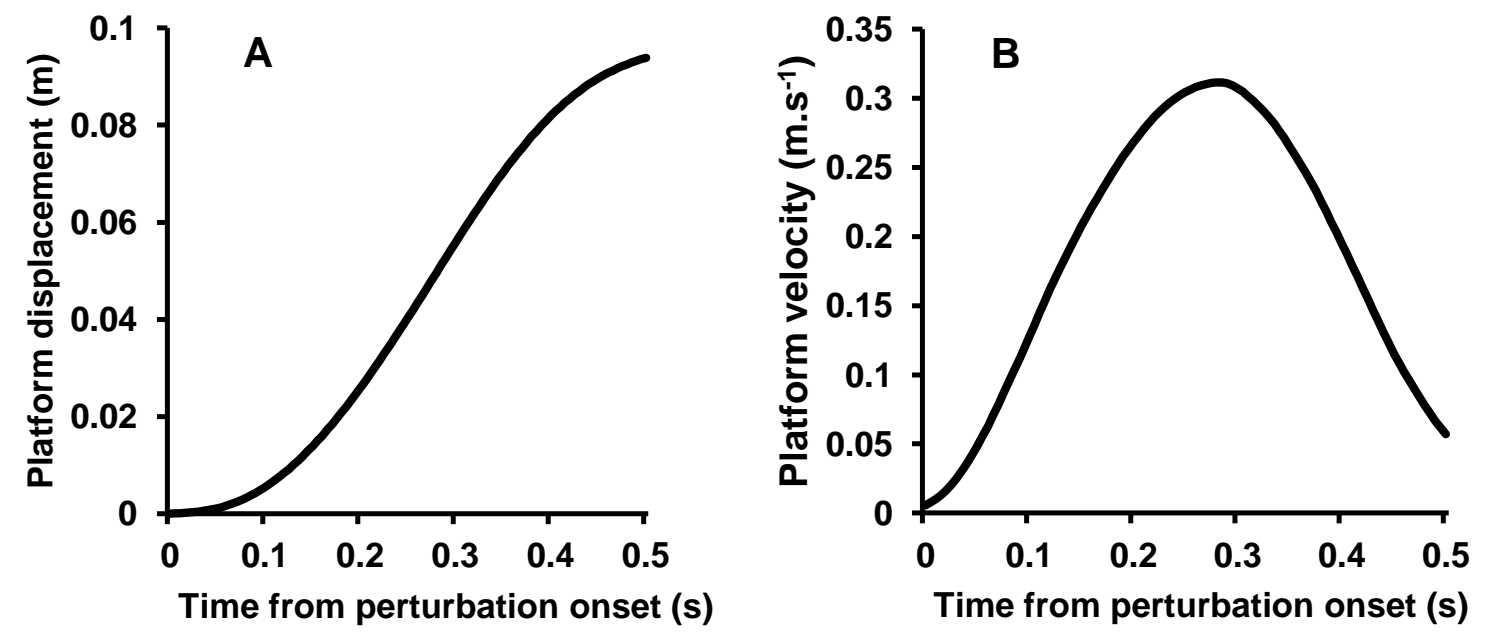

Figure 1. 

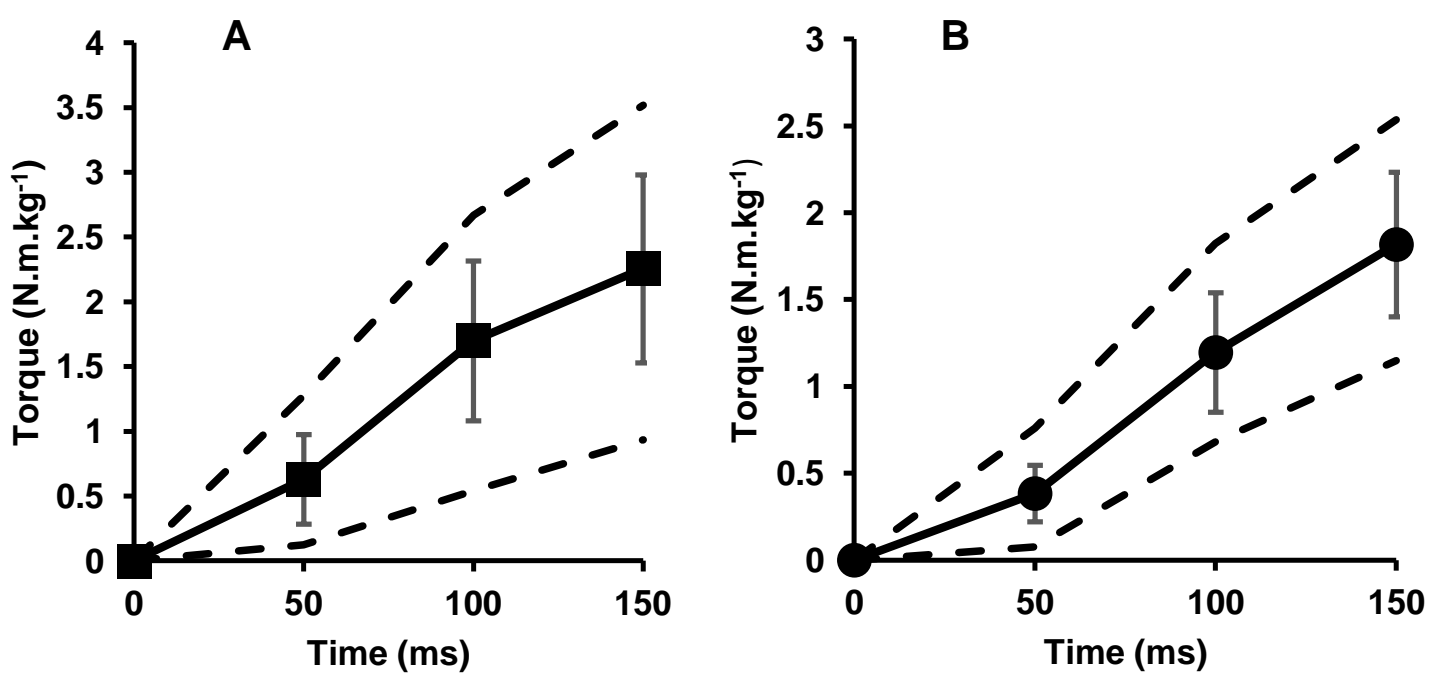

Figure 2. 

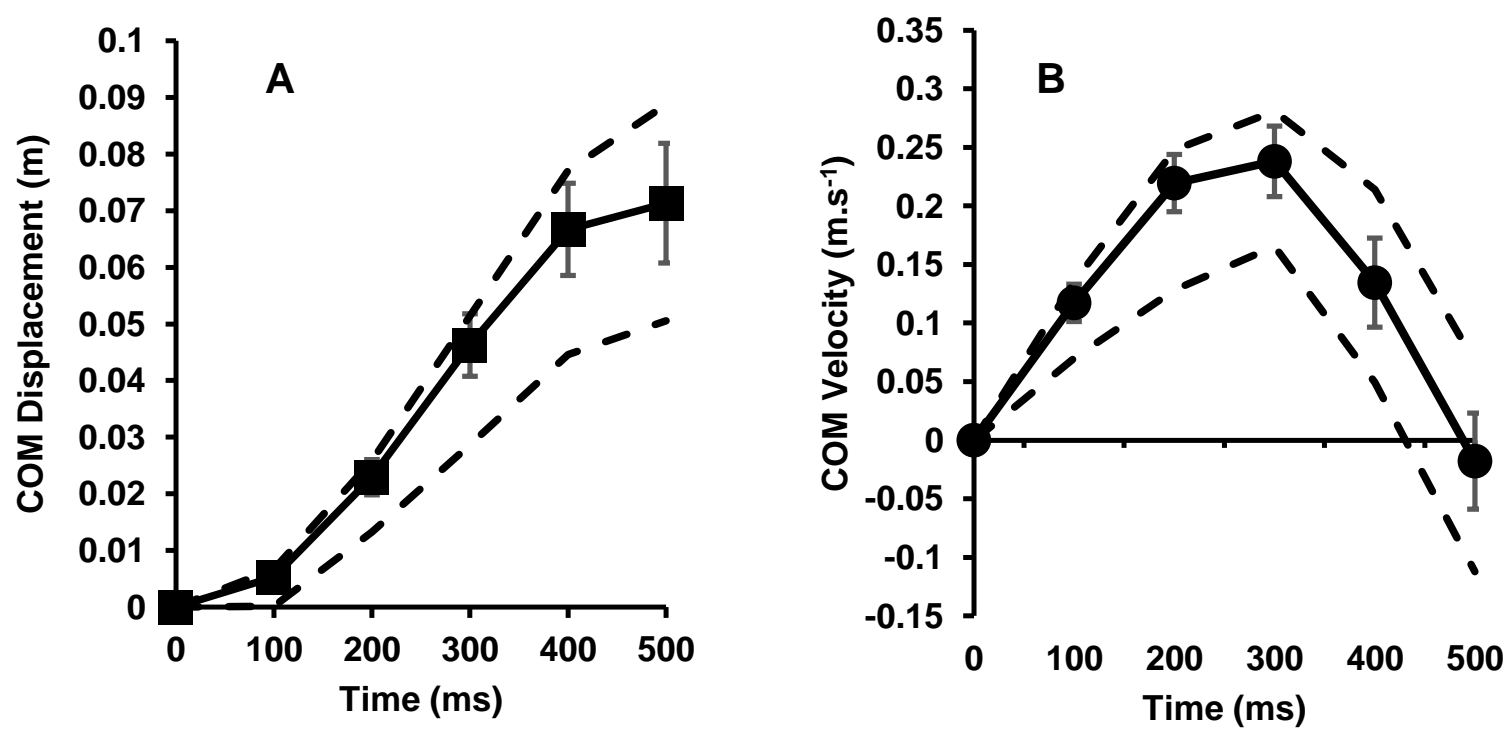

Figure 3. 

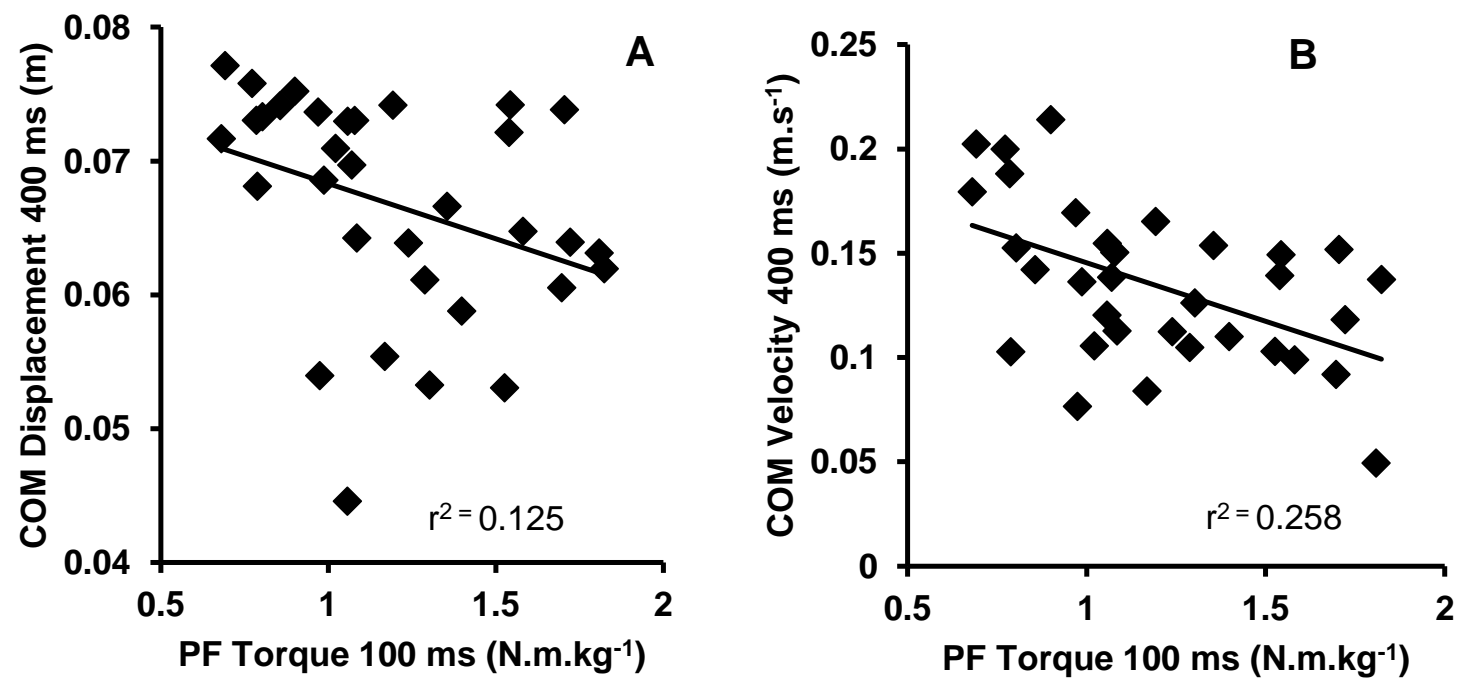

Figure 4. 

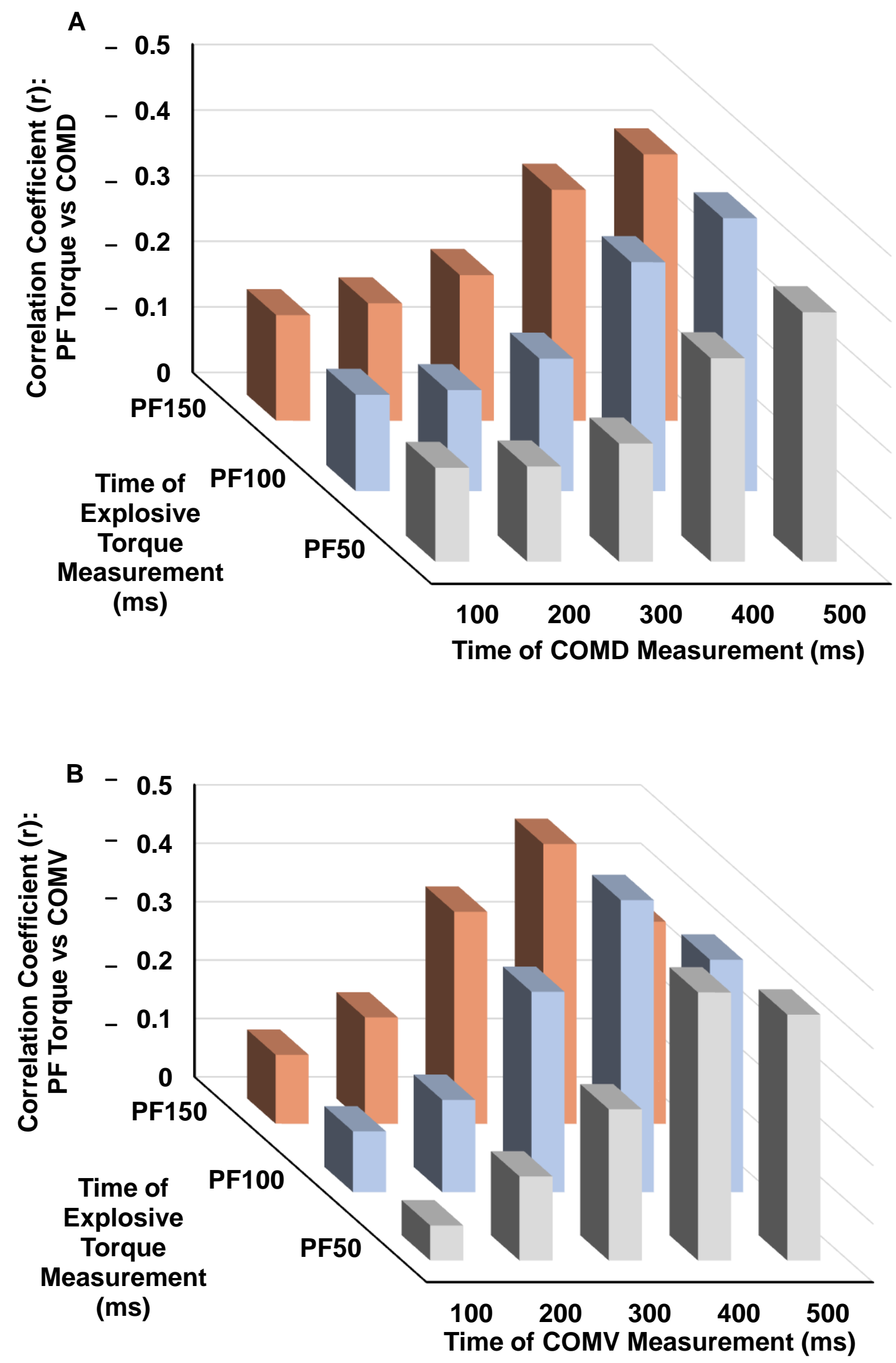

Figure 5. 

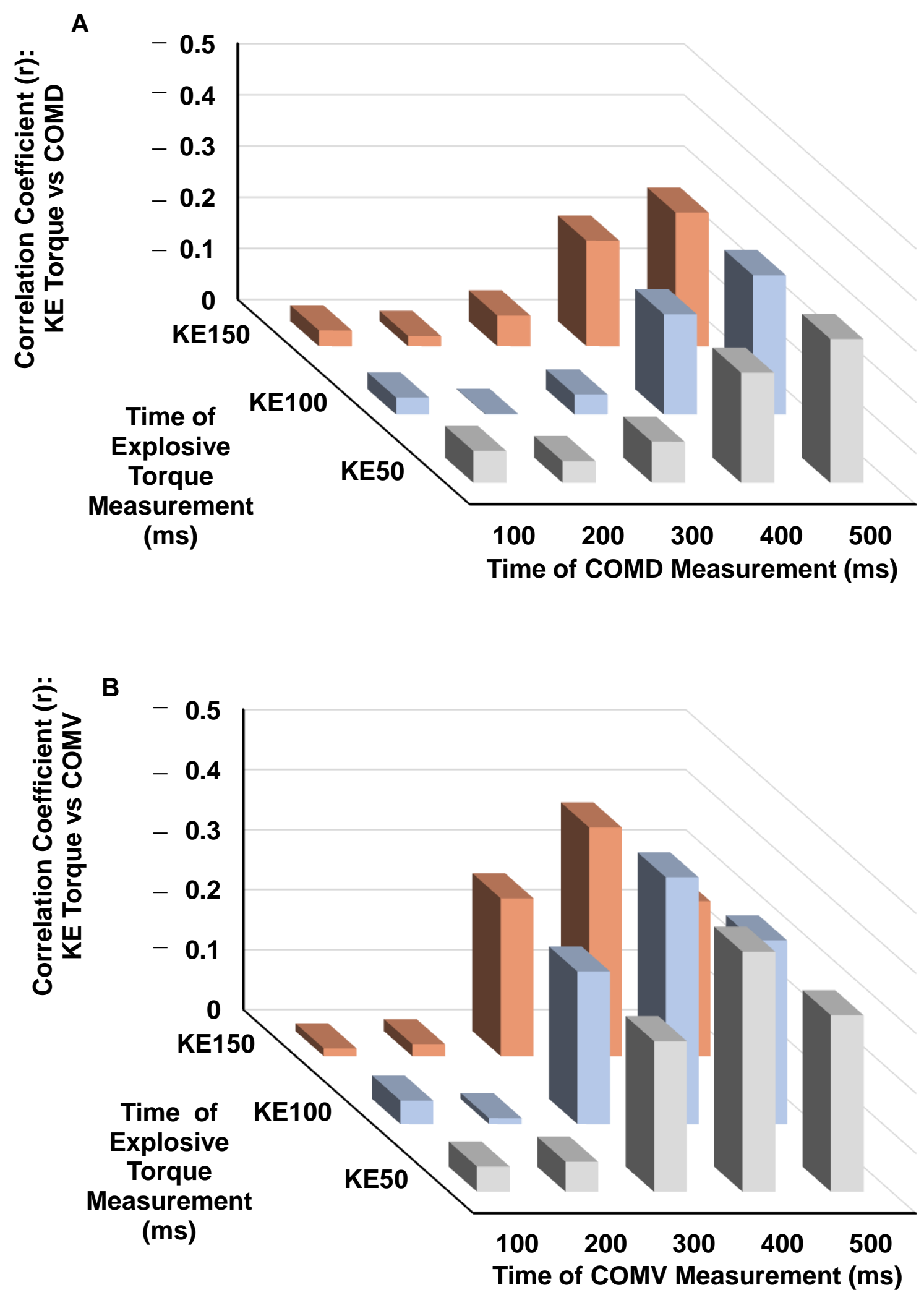

Figure 6. 


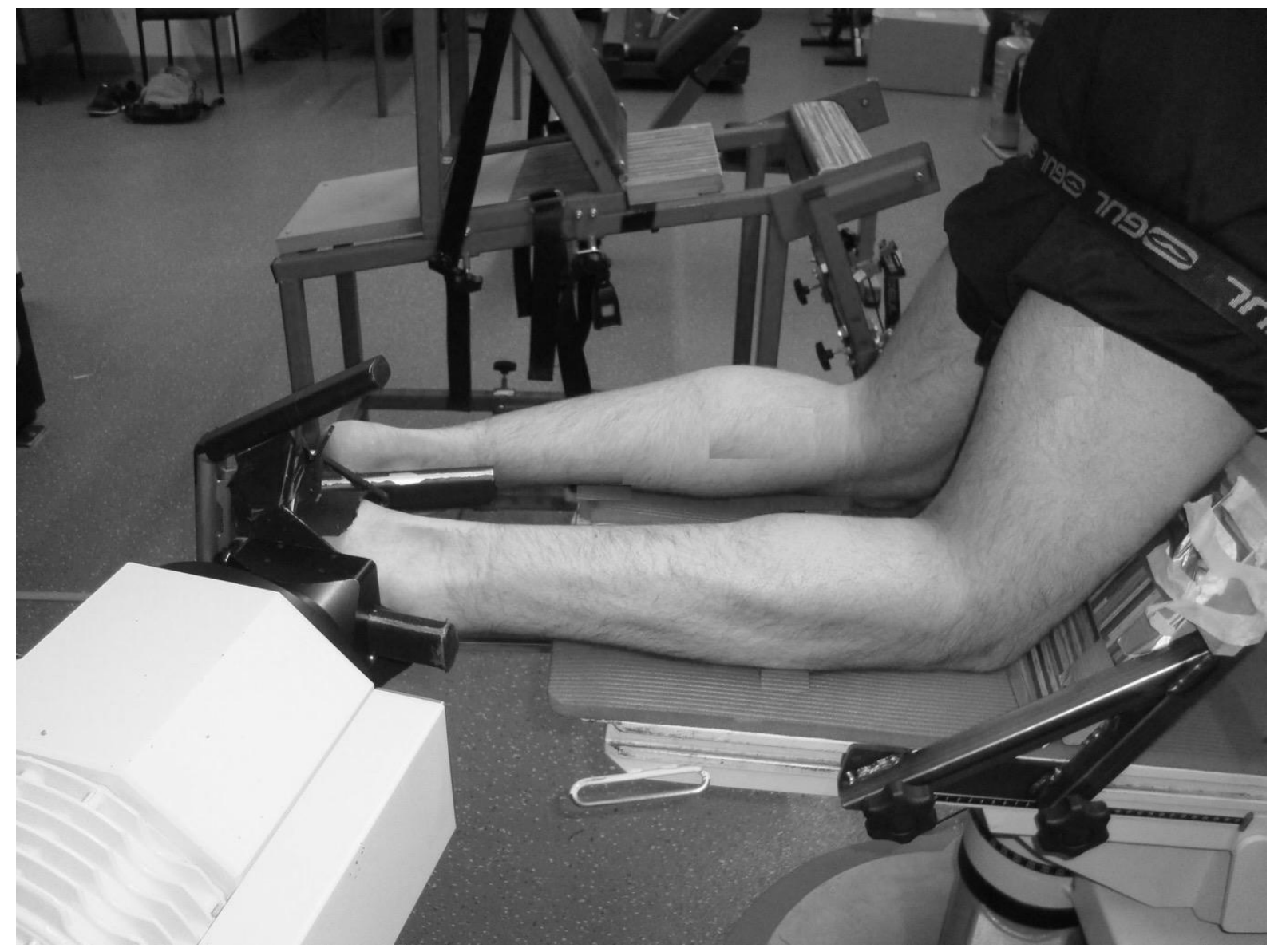

Appendix 1A. Isometric maximum and explosive voluntary torque measurements of the plantar flexors with an adapted Con-trex isokinetic dynamometer. 


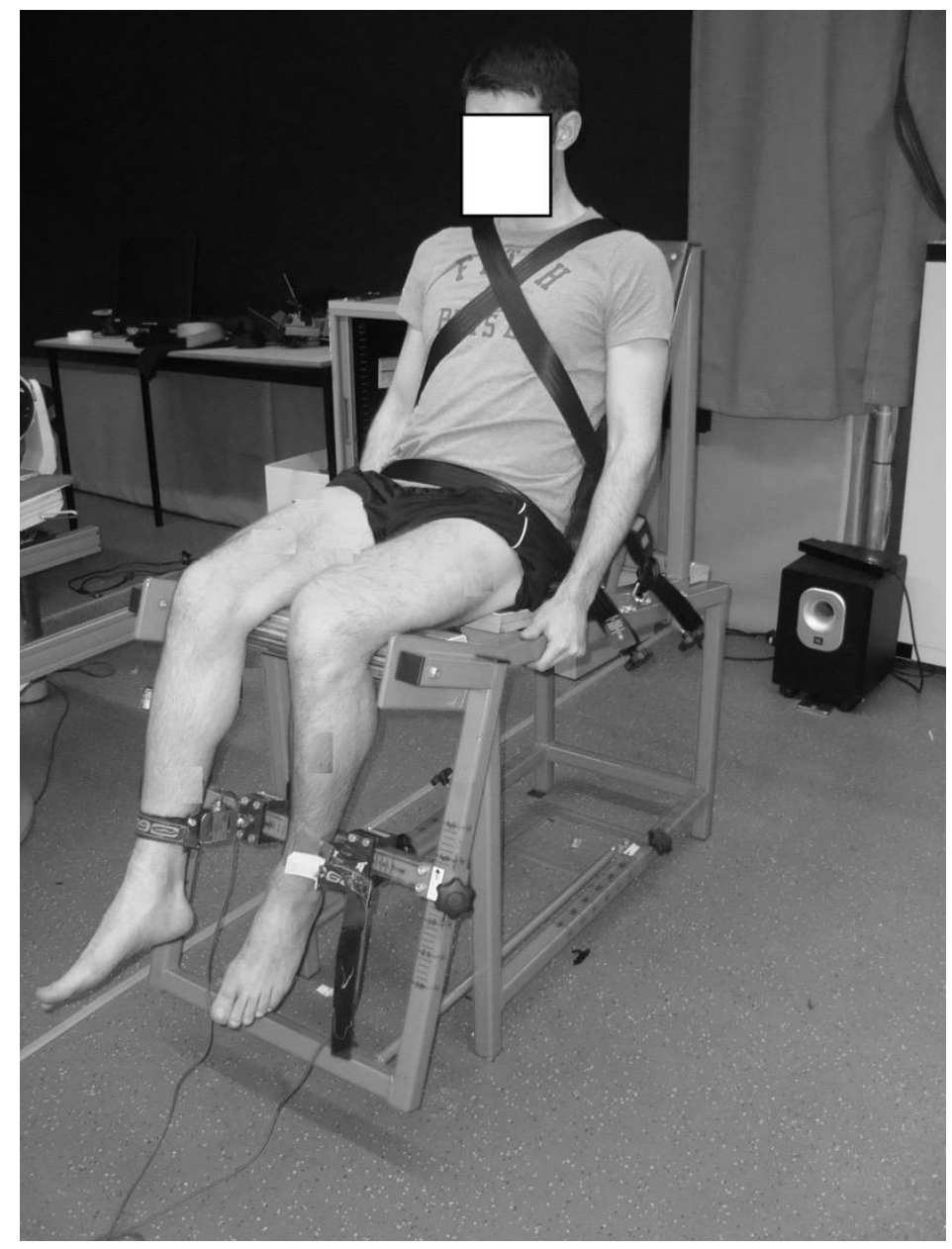

Appendix 1B. Isometric maximum and explosive voluntary torque measurements of the knee extensors with a custom built isometric dynamometer. 